\title{
Position Estimation Error Performance Model for a Minimum Configuration 3-D Multilateration
}

\author{
Abdulmalik Shehu Yaro ${ }^{1}$, Ahmad Zuri Sha'ameri ${ }^{1}$, and Nidal Kamel ${ }^{2}$ \\ ${ }^{1}$ Department of Electrical and Computer Engineering, Universiti Teknologi Malaysia, Johor, Malaysia \\ ${ }^{2}$ Department of Electrical and Electronic Engineering, Universiti Teknologi Petronas, Perak, Malaysia \\ yaroabdulmalik@yahoo.com,zuri@fke.utm.my and nidalkamel@petronals.com.my
}

\begin{abstract}
A multilateration system estimates the position of emitter using time difference of arrival (TDOA) measurements with a lateration algorithm. It involves solving a set of hyperbolic plane equations to determine the position of the emitter given the TDOA measurements that corresponds to the path difference (PD) measurement in distance. A performance model is developed using the relative maximum error bound (RMEB) which relates the plane equation condition number, the relative ground receiving station (GRS) geometry and the PD measurement error to estimate the position estimation (PE) error. By using air traffic monitoring for civil aviation as an application, Monte Carlo simulation verifies the PE error of the performance model for a square GRS configuration. The coverage assumed a $360^{\circ}$ bearing, a range of up to $200 \mathrm{~km}$ and a maximum altitude of $15 \mathrm{~km}$. Simulation results also show that the performance model estimates the horizontal position error with a maximum absolute error of $0.1 \mathrm{~km}$ up to a range of $200 \mathrm{~km}$ at an altitude of 15 $\mathrm{km}$ and a minimum absolute error of $0.2 \mathrm{~km}$ at an altitude of $15 \mathrm{~km}$.
\end{abstract}

Keywords: Multilateration, Time difference of arrival (TDOA), Performance model, least square error bound.

\section{Introduction}

The emitter locating technique used in navigation, air traffic monitoring regulatory body and military consists of a two-stage process [1]. The first stage estimates the position-dependent signal parameters such as time of arrival (TOA), angle of arrival (AOA), time difference of arrival (TDOA) and received signal strength (RSS). The second stage uses the estimated position-dependent signal parameters as input to a localization algorithm such as lateration, angulation, and fingerprinting to determine the location of emitter. Multilateration locates a target from its electronic emission by measuring the TDOAs from a network of spatially-located GRSs. The TDOA measurements form the position-dependent signal parameters $[1,2]$ which are then used by the lateration algorithm to estimate the position of the aircraft $[1,3]$. Depending on the number of GRSs deployed, the position of the aircraft may be estimated either in 2dimension (2-D) $(x, y)$ or 3-dimension (3-D) $(x, y, z)$ [2]. A minimum of four (4) GRSs is required to estimate the $3-\mathrm{D}$ position of an aircraft.

TDOA estimation methods reported in various articles can be grouped as $[1,3,4]$ : indirect and direct. The indirect method involves estimating the TOA of the signal at each GRS and the TDOA is estimated from the difference between a TOA pair. This method can only be used when there is a definite pulse edge of the signal to measure [2]. Several techniques for TOA estimation has been reported [5-8]. The most commonly used technique is the leading-edge detection used for secondary surveillance radar (SSR) signals [7, 8]. In the direct method, the TDOA is estimated directly from the signal received from a GRS pair. The most commonly used method applies the cross-correlation between a GRS pair to estimate the TDOA [2, 9, 10]. Other direct methods for TDOA estimation are generalized cross-correlation (GCC) [11], high-order statistic (HOS) [10], least mean squares (LMS) adaptive filter [10], average squared difference function (ASDF) and average magnitude difference function (AMDF) [10]. Direct methods operate directly on the signal without any knowledge of the signal parameter o structure. Thus, a

Received: July $11^{\text {st }}$ 2017. Accepted: March $29^{\text {th }}, 2018$

DOI: $10.15676 /$ ijeei.2018.10.1.11 
sufficiently large bandwidth and reliable data link are required between the GRSs to reach the processing center. Otherwise, the indirect method is preferable [2].

From the estimated TDOA and GRS positions, the second as well as the final stage is the position estimation (PE) of the emitter using the lateration algorithm. $N$ numbers of GRSs with a single GRS as reference results in $N-1$ nonlinear hyperbolic equations that describe the TDOA measurement input and the position of emitter [12]. Due to this nonlinearity, several methods are developed to solve these equations [12-25] either in linear/closed-form method or nonlinear/open-form method $[1,12]$. In the nonlinear method, the first step is to linearize the nonlinear hyperbolic equations using series expansion such as the Taylor series expansion method [13-15]. An initial random position of emitter is first inputted and iteratively refined to the final position estimate by minimizing the least square (LS) cost function. The nonlinear approach has limitations for real-time application since the PE solution convergence depends on the difference between the initial PE estimates with the actual position of emitter [15]. For the linear method, the algebraic manipulation of a pair of hyperbolic equations results in a single linear plane equation with the position of emitter as the unknown variable [16-22]. The resulting plane equation is expressed as:

$$
x B+y C+z D=A
$$

The result where the coefficients $A, B, C$, and $D$ depends on TDOA estimates and GRS coordinates. The linear approach has no convergence issue since it does not require the estimate of the initial position of emitter [15]. It is faster than the nonlinear method but is very sensitive to the input measurement error.

Most of the published work emphasized on reducing the PE error of the lateration algorithm at high TDOA estimation error [17, 20,23-25] or on performance analysis [18, 19]. The use of multiple GRSs as references provides further improvement on the PE accuracy of emitter [17, 23]. However, this paper focused on a performance model to predict the PE error for a multilateration system. Application based on air traffic monitoring for civil aviation verifies the performance model. Multilateration is a surveillance technology that complements existing systems such as primary surveillance radar (PSR), SSR and automatic surveillance- dependentbroadcast (ADS-B) [26, 27]. Documents from international bodies such as the International Civil Aviation Organization (ICAO) [4] or manufacturers [27-29] only specify the PE error but not the range, and the link with the transmitter and receiver parameters such as transmit power, sensitivity and antenna gain. Therefore, the proposed performance model allows a systematic approach to predict the coverage and plan for future deployment of multilateration system.

Multilateration estimates emitter position using the geometrical relationship between the GRS configuration and the emitter [30]. This means the accuracy in the PE depends on the GRS configuration. Several studies are carried out on the best GRS configuration for high PE accuracy $[14,30-32]$. For a few GRSs of 4 or less, simple configurations such as squares and equilateral triangles result in better PE accuracy. The minimum number of GRS for 3-D PE with multilateration is 4. It was shown by Chan et al. [31] that square configuration of four GRSs performances better than the collinear GRS configurations. Since the four GRSs are considered in this work, the square GRS configuration is adopted.

A linear multiple reference lateration algorithm is developed for a 3-D multilateration system with a square GRS configuration. Based on the relative maximum error bound (RMEB) and linear regression, the performance model is developed to predict the PE error using the plane equation condition number, GRS coordinates, transmitter parameters, and receiver parameters. The PE error comparison between the performance model and the Monte Carlo simulation assumed a square GRS configuration with a GRS pair separation of $10 \mathrm{~km}$.

The organisation of the paper is as follows. Section 2 describes the sources of error and the type of signals received by the multilateration system. The PE methodology is introduced in section 3, while the proposed methodology for PE error is derived in section 4. Simulation results and discussion based on a frequency band of $1090 \mathrm{MHz}$, which are used by the SSR and ADS-B for air traffic monitoring in civil aviation is presented in section 5, followed by the conclusion. 


\section{Multilateration Signals and Error}

The target application for the multilateration system is for air traffic monitoring in civil aviation since the position of aircraft is represented in 3-D: latitude, longitude, and altitude. Besides communication purposes, the electronic emission produced by an aircraft is also used for PE such as ADS-B, SSR, weather radar and weapon control radar [4, 18]. The ADS-B and SSR operate at $1090 \mathrm{MHz}$ band while weather radar and weapon control radar operate in the Xband $(8-12 \mathrm{GHz})$ [2]. For air traffic monitoring, the multilateration system operates on the ADS$B$ and SSR to locate the aircraft.

The accuracy in the PE primarily depends on the accuracy of the TDOA estimate [33], the number of GRSs and their configurations [34]. In addition, the sources of error are natural errors and system errors [33]. Natural errors include noise floor due to thermal noise while path loss attenuation and multipath fading affect the actual signal-to-noise ratio (SNR) of the signal. System error is mostly related to system hardware including quantization error and antenna error. In this research, the major contributor to the TDOA measurement error is the thermal noise modelled as additive white Gaussian noise (AWGN), quantified by the SNR.

\section{Position Estimation Methodology}

This section first describes the linear method lateration algorithm using two references of GRS followed by a discussion on the location of blind spots in the PE process.

\section{A. Two Reference Lateration Algorithm}

Given the position of an emitter in the Cartesian coordinate $[x, y, z]^{T}$ and the position of the $i$-th GRS as $\left[x_{i}, y_{i}, z_{i}\right]^{T}$ for $i=1,2,3$, and 4, the Euclidean distance (path distance) between the emitter and the $i$-th GRS is calculated as:

$$
d_{i}=c \times \tau_{i}=\sqrt{\left(x-x_{i}\right)^{2}+\left(y-y_{i}\right)^{2}+\left(y-y_{i}\right)^{2}}
$$

where $c$ is the speed of light at $3 \times 10^{8} \mathrm{~m} / \mathrm{s}$ and $\tau_{i}$ is the TOA at the $i$-th GRS.

Using Eq. (2), the path difference (PD) between the $i$-th and the $j$-th GRS pair is given as:

$$
\begin{aligned}
d_{i j} & =d_{i}-d_{j}=c \times\left(\tau_{i}-\tau_{j}\right) \\
& =\sqrt{\left(x-x_{i}\right)^{2}+\left(y-y_{i}\right)^{2}+\left(z-z_{i}\right)^{2}}-\sqrt{\left(x-x_{j}\right)^{2}+\left(y-y_{j}\right)^{2}+\left(z-z_{j}\right)^{2}}
\end{aligned}
$$

For simplicity, using GRS 1 and GRS 2 as a reference pair for TDOA estimation [22] and $d_{i j}=-d_{i j}$, the 4 PD equations are given as:

$$
\begin{aligned}
& d_{13}=d_{1}-d_{3} \\
& d_{14}=d_{1}-d_{4} \\
& d_{23}=d_{2}-d_{3} \\
& d_{24}=d_{2}-d_{4}
\end{aligned}
$$

Substituting Eq. (3) into Eq. (4) results in two plane equations in the form

$$
\begin{aligned}
& A_{134}=x B_{134}+y C_{134}+z D_{134} \\
& A_{234}=x B_{234}+y C_{234}+z D_{234}
\end{aligned}
$$

where the coefficients of Eq. (5) and Eq. (6) are 


$$
\begin{aligned}
& A_{i 34}=0.5\left(d_{i 4}-d_{i 3}+\frac{K_{i 4}}{d_{i 4}}-\frac{K_{i 3}}{d_{i 3}}\right) \\
& B_{i 34}=\left(\frac{X_{3 i}}{d_{i 3}}-\frac{X_{4 i}}{d_{i 4}}\right) \\
& C_{i 34}=\left(\frac{Y_{3 i}}{d_{i 3}}-\frac{Y_{4 i}}{d_{i 4}}\right) \\
& D_{i 34}=\left(\frac{Z_{3 i}}{d_{i 3}}-\frac{Z_{4 i}}{d_{i 4}}\right) \\
& X_{j i}=x_{j}-x_{i}, Y_{j i}=y_{j}-y_{i}, Z_{j i}=z_{j}-z_{i} \\
& K_{i 3}=\left(x_{i}^{2}+y_{i}^{2}+z_{i}^{2}\right)-\left(x_{3}^{2}+y_{3}^{2}+z_{3}^{2}\right) \\
& K_{i 4}=\left(x_{i}^{2}+y_{i}^{2}+z_{i}^{2}\right)-\left(x_{4}^{2}+y_{4}^{2}+z_{4}^{2}\right)
\end{aligned}
$$

for $i \epsilon[1,2]$ and $j \epsilon[3,4]$ or

Rearranging Eq. (5) and Eq. (6) in matrix form results in

$$
\left[\begin{array}{lll}
B_{134} & C_{134} & D_{134} \\
B_{234} & C_{234} & D_{234}
\end{array}\right] \times\left[\begin{array}{l}
x \\
y \\
z
\end{array}\right]=\left[\begin{array}{l}
A_{134} \\
A_{234}
\end{array}\right]
$$

The derivation details from Eq. (4) to Eq. (8) can be found in $[1,3,18,22]$ and are not included in this paper due to complexity. Eq. (8) is an underdetermined least square (LS) problem where no unique solution exists [35]. If $N \geq 5$ GRSs, the matrix Eq. (8) will be fullranked or over-determined and can be solved using LS method. Assuming the GRS height differences are negligible

$$
Z_{i j}=z_{i}-z_{j} \approx 0 \text { for } i \epsilon[1,2] \text { and } j \epsilon[3,4]
$$

The coefficients of $z$ from Eq. (5) and Eq. (6) become zero, that is $D_{134} \approx D_{234} \approx 0$.

Rewriting the matrix Eq. (8) results in

$$
\begin{aligned}
& {\left[\begin{array}{ll}
B_{134} & C_{134} \\
B_{234} & C_{234}
\end{array}\right] \times\left[\begin{array}{l}
x \\
y
\end{array}\right]=\left[\begin{array}{l}
A_{134} \\
A_{234}
\end{array}\right]} \\
& \mathbf{Q x}=\mathbf{a}
\end{aligned}
$$

The horizontal position $(x, y)$ obtained by matrix inversion in Eq. (10) is substituted back into Eq. (4a) producing

$$
\left(\frac{d_{13}}{2}+x \times \frac{X_{31}}{d_{13}}+y \times \frac{Y_{31}}{d_{13}}+\frac{K_{13}}{2 d_{13}}\right)^{2}=\left(x-x_{1}\right)^{2}+\left(y-y_{1}\right)^{2}+\left(z-z_{1}\right)^{2}
$$

By simplifying Eq. (11) as a quadratic equation, the altitude is estimated by solving

$$
z=z_{1}+\sqrt{w+\left(x-x_{1}\right)^{2}+\left(y-z_{1}\right)^{2}}
$$


where

$$
w=\left(0.5 d_{13}+x \frac{\left(x_{3}-x_{1}\right)}{d_{13}}+y \frac{\left(y_{3}-y_{1}\right)}{d_{13}}+\frac{K_{13}}{d_{13}}\right)^{2}
$$

In surveillance system for air traffic monitoring, target locations are defined in the cylindrical coordinate system $(R, \theta, z)$ where $R$ is the horizontal range of the target taken from the reference point, $\theta$ is the bearing in degree and $z$ is the target altitude taken from the sea level [36]. The following equation converts cylindrical coordinate system to Cartesian coordinate system

$$
\begin{aligned}
& x=R \times \cos (\theta) \\
& y=R \times \sin (\theta)
\end{aligned}
$$

\section{B. Position Estimation Process Blind Spots}

For a given GRS configuration, blind spots occur when the PD estimated between a GRS pair is zero. As a result, some of the variables in Eq. (7) become infinite, making it impossible to use Eq. (10) to solve the position of emitter. Given $i$-th and $j$-th GRSs at position $\left(x_{i}, y_{i}, z_{i}\right)$ and $\left(x_{j}, y_{j}, z_{j}\right)$ respectively, a blind spot exits if

$$
d_{i j}=d_{i}-d_{j}=0
$$

To obtain the mathematical representation of the blind spot in range $(R)$ and bearing $(\theta)$, substituting Eq. (15) into Eq. (3) results in

$$
\Psi(R, \theta, z)=R \times\left(X_{j i} \cos \theta+Y_{j i} \sin \theta\right)+z \times Z_{j i}-0.5 K_{j i}
$$

where the coefficients of Eq. (16) are

$$
\begin{aligned}
& X_{j i}=x_{j}-x_{i} \\
& Y_{j i}=y_{j}-y_{i} \\
& Z_{j i}=z_{j}-z_{i} \\
& K_{i j}=\left(x_{i}^{2}+y_{i}^{2}+z_{i}^{2}\right)-\left(x_{j}^{2}+y_{j}^{2}+z_{j}^{2}\right)
\end{aligned}
$$

Using the GRS position pair of $\left(x_{i}, y_{i}, z_{i}\right)$ and $\left(x_{j}, y_{j}, z_{j}\right)$, the target range $R$ and bearing $\theta$ that makes Eq. (16) zero value is the blind spot. The location of blind spot varies with the choice of the GRS reference pair.

\section{Proposed Position Estimation Error Methodology}

The PE methodology described in section 3 as shown in Figure 1 consists of two stages: horizontal PE error and vertical PE error. The performance model provides the PE error for a given position of emitter, position of GRS and path difference estimation (PDE) error.

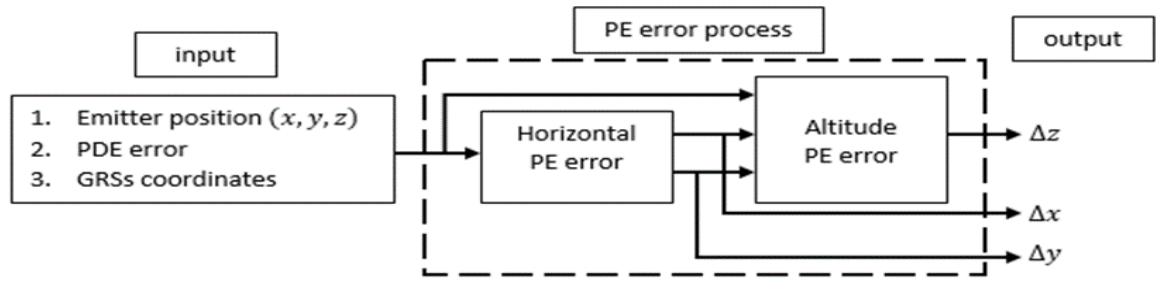

Figure 1. Position estimation error process. [ $\Delta \mathrm{x}$ is the error in $\mathrm{x}$ coordinate, $\Delta \mathrm{y}$ is the error in $\mathrm{y}$ coordinate and $\Delta \mathrm{z}$ is the error in $\mathrm{z}$ coordinate]. 


\section{A. Horizontal PE Error}

The error in the horizontal position (x) obtained from Eq. (10) is due to the TDOA measurement error that is proportional to the PDE error in distance. This is reflected by the sensitivity of matrix $\mathrm{Q}$ from the plane equation in Eq. (10) which is known as the plane equation condition number denoted by $K(\mathbf{Q})$. The RMEB defined by Eq. (18) relates the condition number, the relative emitter horizontal PE error matrix, and the relative GRS geometry and the PD measurement matrix error [35].

$$
\frac{\|\Delta \mathbf{x}\|_{2}}{\|\mathbf{x}\|_{2}} \leq K(\mathbf{Q}) \times\left(\frac{\|\Delta \mathbf{Q}\|_{2}}{\|\mathbf{Q}\|_{2}}+\frac{\|\Delta \mathbf{a}\|_{2}}{\|\mathbf{a}\|_{2}}\right)
$$

where $\Delta \mathbf{x}=[\Delta x, \Delta y]$ is the emitter horizontal PE error, $\frac{\|\Delta \mathbf{x}\|_{2}}{\|\mathbf{x}\|_{2}}$ is the relative emitter horizontal PE error, $\left(\frac{\|\Delta \mathbf{Q}\|_{2}}{\|\mathbf{Q}\|_{2}}+\frac{\|\Delta \mathbf{a}\|_{2}}{\|\mathbf{a}\|_{2}}\right)$ is the relative input error matrix which is related to the position of GRS and PD measurement from Eq. (7), and \|\|$_{2}$ is the $\mathrm{L}_{2}$ matrix norm which is also known as the spectral norm. Detailed descriptions of $L_{2}$ matrix norm and $L_{2}$ vector norm can be found in [39]. The proposed performance model to determine the horizontal PE error is derived by transforming (18) into a representation that is a function of the plane equation condition number $K(\mathbf{Q})$, the position of emitter $(R, \theta, z)$, GRS positions $\left(x_{i}, y_{i}, z_{i}\right)$ and PDE error $(\Delta d)$. Linear regression curve fitting is used for this purpose and the procedure of estimating the horizontal PE error $\Delta \mathbf{x}$ initiated by finding the plane equation condition number $K(\mathbf{Q})$ for each position of emitter $(x, y, z)$. The next step is deriving the relative GRS geometry and PD matrix error $\left(\frac{\|\Delta \mathbf{Q}\|_{2}}{\|\mathbf{Q}\|_{2}}+\frac{\|\Delta \mathbf{a}\|_{2}}{\|\mathbf{a}\|_{2}}\right)$ as a function of PDE error $(\Delta d)$ and PD measurement matrix (see Appendix A). Consequently, the resulting function is expressed as follows:

$$
\left(\frac{\|\Delta \mathbf{Q}\|_{2}}{\|\mathbf{Q}\|_{2}}+\frac{\|\Delta \mathbf{a}\|_{2}}{\|\mathbf{a}\|_{2}}\right)=\Delta d \times\left\|\left[\begin{array}{cc}
d_{13}^{-2} & d_{14}^{-2} \\
d_{24}^{-2} & d_{23}^{-2}
\end{array}\right]\right\|_{2} \times\left\|\left[\begin{array}{cc}
d_{13}^{-1} & d_{14}^{-1} \\
d_{24}^{-1} & d_{23}^{-1}
\end{array}\right]^{-1}\right\|_{2}
$$

By substituting Eq. (19) into the of Eq. (18), the RMEB is now

$$
R_{\text {RMEB }}=K(\mathbf{Q}) \times \Delta d \times\left\|\left[\begin{array}{ll}
d_{13}^{-2} & d_{14}^{-2} \\
d_{24}^{-2} & d_{23}^{-2}
\end{array}\right]\right\|_{2} \times\left\|\left[\begin{array}{ll}
d_{13}{ }^{-1} & d_{14}{ }^{-1} \\
d_{24}{ }^{-1} & d_{23}{ }^{-1}
\end{array}\right]^{-1}\right\|_{2}
$$

The main objective here is to use $R_{R M E B}$ to predict the performance of the multilateration system using Eq. (20). A correction factor $\alpha$ is introduced to correlate the measured relative horizontal PE error and the relative horizontal PE error $\left(R_{R M E B}\right)$ from Eq. (20). This correction factor depends primarily on the emitter bearing relative to the center of the GRS configuration. For a given position of emitter with a bearing of $\theta$, the measure horizontal PE error, $R_{A C}$ is

$$
R_{A C} \approx \alpha(\theta) \times R_{R M E B} \text { for } 0 \leq \theta \leq 360^{\circ}
$$

Using linear regression, the value of the correction factor $\alpha$ for emitters with a bearing of $0^{0}$ and $360^{\circ}$ can be determined by minimizing the error defined by Eq. (22) using linear regression

$$
E=\underset{\alpha(\theta)}{\arg \min }\left\|\left(R_{A C}-\alpha(\theta) \times R_{R M E B}\right)\right\|_{2}
$$

Taking the derivative of Eq. (22) with respect to $\alpha$ and solve for $\partial E / \partial \alpha=0$, results in

$$
\alpha(\theta)=\frac{\left\|R_{A C} \times R_{R M E B}\right\|_{2}}{\left\|\left(R_{R M E B}\right)^{2}\right\|_{2}}
$$

The resulting expression for $\alpha(\theta)$ in Eq. (23) for a square GRS configuration is:

$$
\begin{aligned}
\alpha(\theta) & =-2.768 \times(|\sin (\theta)|)^{4}+2.598 \times(|\sin (\theta)|)^{3}-0.432 \times(|\sin (\theta)|)^{2} \\
& +0.6697 \times(|\sin (\theta)|)+0.013
\end{aligned}
$$


Since the error is minimal, the relative horizontal PE error $\left(\frac{\left\|\Delta \mathbf{x}_{p m}\right\|_{2}}{\|\mathbf{x}\|_{2}}\right)$ for the performance model can now be expressed as:

$$
\frac{\left\|\Delta \mathbf{x}_{p m}\right\|_{2}}{\|\mathbf{x}\|_{2}}=\alpha(\theta) \times k(\mathbf{Q}) \times \Delta d \times\left\|\left[\begin{array}{ll}
d_{13}^{-2} & d_{14}^{-2} \\
d_{24}^{-2} & d_{23}^{-2}
\end{array}\right]\right\|_{2} \times\left\|\left[\begin{array}{ll}
d_{13}{ }^{-1} & d_{14}{ }^{-1} \\
d_{24}{ }^{-1} & d_{23}{ }^{-1}
\end{array}\right]^{-1}\right\|_{2}
$$

From Eq. (25) the horizontal PE error for the performance model is expressed as:

$$
\left\|\Delta \mathbf{x}_{p m}\right\|_{2}=\alpha(\theta) \times k(\mathbf{Q}) \times \Delta d \times\left\|\left[\begin{array}{ll}
d_{13}^{-2} & d_{14}^{-2} \\
d_{24}^{-2} & d_{23}^{-2}
\end{array}\right]\right\|_{2} \times\left\|\left[\begin{array}{ll}
d_{13}{ }^{-1} & d_{14}{ }^{-1} \\
d_{24}{ }^{-1} & d_{23}{ }^{-1}
\end{array}\right]^{-1}\right\|_{2} \times\left\|_{2}\right\|_{2}
$$

Eq. (26) is the performance model for the horizontal PE error for multilateration. Using vector analysis, the components of the horizontal PE error $(\Delta \mathbf{x})$ obtained from the position of emitter $(x, y)$ are

$$
\begin{aligned}
& \Delta x=\frac{x}{x+y} \times\left\|\Delta \mathbf{x}_{p m}\right\|_{2} \\
& \Delta y=\frac{y}{x+y} \times\left\|\Delta \mathbf{x}_{p m}\right\|_{2}
\end{aligned}
$$

Finally, the components of the estimated horizontal position $(\hat{\mathbf{x}})$ are

$$
\hat{\mathbf{x}}=[\hat{x}, \hat{y}]
$$

where $\hat{x}=x+\Delta x$ and $\hat{y}=y+\Delta y$

The estimated horizontal position $\hat{\mathbf{x}}$ is used together with $\Delta d$ to estimate the altitude error $\Delta z$ in the next section.

\section{B. Altitude Estimation Error}

The estimated horizontal position in Eq. (28) is used to obtain the altitude error by substituting Eq. (27) and $\Delta d$ into Eq. (12). The estimated altitude of emitter is

$$
\hat{z}=z_{1}+\sqrt{\hat{w}+\left(\hat{x}-x_{1}\right)^{2}+\left(\hat{y}-y_{1}\right)}
$$

where

$$
\begin{aligned}
& \hat{w}=\left(0.5 \hat{d}_{13}+\hat{x} \frac{\left(x_{3}-x_{1}\right)}{\hat{d}_{13}}+\hat{y} \frac{\left(y_{3}-y_{1}\right)}{\hat{d}_{13}}+\frac{K_{13}}{\hat{d}_{13}}\right)^{2} \\
& \hat{d}_{13}=d_{13}+N(0, \Delta d) \\
& K_{13}=\left(x_{1}^{2}-x_{3}^{2}\right)+\left(y_{1}^{2}-y_{3}^{2}\right)+\left(z_{1}^{2}-z_{3}^{2}\right)
\end{aligned}
$$

To obtain the altitude error $(\Delta z)$, the result from Eq. (29) is subtracted from a known altitude of emitter, $\Delta z=\hat{z}-z$ and the altitude error for the performance model is

$$
\Delta z=\left|\left(z_{1}+\sqrt{\hat{w}+\left(\hat{x}-x_{1}\right)^{2}+\left(\hat{y}-y_{1}\right)}\right)-z\right|
$$

A performance model for predicting PE error of a 3-D position of emitter using multilateration system based on the minimum GRS configuration is derived. Next section validates the performance model by comparing with the Monte Carlo simulation. 


\section{Simulation Parameters, Results and Discussion}

This section first presents the simulation parameters followed by the comparison between the developed performance model and the Monte Carlo simulation.

\section{A. Simulation Parameter}

The root-mean-square error (RMSE) of the position is used as the performance measure to compare the result of performance model with that of Monte Carlo simulation. For $N$-realization Monte Carlo simulation, the following equations define the RMSE of the horizontal position and altitude

$$
H_{\text {rmse }}=\sqrt{\frac{\sum_{i=1}^{N}\left[\left(\hat{x}_{i}-x\right)^{2}+\left(\hat{y}_{i}-y\right)^{2}\right]}{N}}, A l t_{\text {rmse }}=\sqrt{\frac{\sum_{i=1}^{N}\left(\hat{z}_{i}-z\right)^{2}}{N}}
$$

where $(x, y, z)$ is the known position of emitter while $\left(\hat{x}_{i}, \hat{y}_{i}, \hat{z}_{i}\right)$ is the estimated position of emitter at the $i$-th Monte Carlo realization.

Table 1 shows the parameters on the position of emitter in terms of range, bearing, and altitude. The 3-D emitter PE is possible if all four GRSs are at line of sight (LOS) with the emitter. The LOS very much depends on the curvature of the earth. For example, the LOS at an altitude of $1 \mathrm{~km}$ is available for all GRSs with a range of up to $130 \mathrm{~km}$ while at $7 \mathrm{~km}$ and $15 \mathrm{~km}$, LOS is available up to a range of $200 \mathrm{~km}$.

Table 1. Emitter position parameters.

\begin{tabular}{|c|c|c|}
\hline No. & Emitter parameters & Values \\
\hline 1 & Horizontal range & $1 \mathrm{~km}$ to $200 \mathrm{~km}$ \\
\hline 2 & Altitude & $1 \mathrm{~km}, 7 \mathrm{~km}$ and $15 \mathrm{~km}$ \\
\hline 3 & Bearing & $0^{0}$ to $359^{0}$ \\
\hline
\end{tabular}

The GRSs are arranged in a square configuration and the separations of 4 different GRS pairs are considered. Table 2 describes the position of each GRS at a separation of $5 \mathrm{~km}, 10 \mathrm{~km}, 20 \mathrm{~km}$ and $30 \mathrm{~km}$.

Table 2. Position of each GRS for various GRS pair separation.

\begin{tabular}{|c|c|c|c|c|c|c|c|c|c|}
\hline \multirow{2}{*}{ No } & \multirow{2}{*}{ GRS label } & \multicolumn{2}{|c|}{$\begin{array}{c}5 \mathrm{~km} \\
\text { separation }\end{array}$} & \multicolumn{2}{c|}{$\begin{array}{c}10 \mathrm{~km} \\
\text { separation }\end{array}$} & \multicolumn{2}{c|}{$\begin{array}{c}20 \mathrm{~km} \\
\text { Separation }\end{array}$} & \multicolumn{2}{c|}{$\begin{array}{c}30 \mathrm{~km} \\
\text { Separation }\end{array}$} \\
\cline { 3 - 11 } & & $\mathrm{x}(\mathrm{km})$ & $\mathrm{y}(\mathrm{km})$ & $\mathrm{x}(\mathrm{km})$ & $\mathrm{y}(\mathrm{km})$ & $\mathrm{x}(\mathrm{km})$ & $\mathrm{y}(\mathrm{km})$ & $\mathrm{x}(\mathrm{km})$ & $\mathrm{y}(\mathrm{km})$ \\
\hline 1 & GRS 1 & -2.5 & 2.5 & -5 & 5 & -10 & 10 & -15 & 15 \\
\hline 2 & GRS 2 & 2.5 & -2.5 & 5 & -5 & 10 & -10 & 15 & -15 \\
\hline 3 & GRS 3 & 2.5 & 2.5 & 5 & 5 & 10 & 10 & 15 & 15 \\
\hline 4 & GRS 4 & -2.5 & -2.5 & -5 & -5 & -10 & -10 & -15 & -15 \\
\hline
\end{tabular}

The PE performance is evaluated based the SSR and ADS-B applications used in the civil aviation and the simulation parameters are shown in Table 3. A transponder that transmits a power of 250 Watts is selected as it corresponds to the type of transponder used on board aircraft capable of flying at an altitude of 15,000 feet $(4.6 \mathrm{~km})$ and above [37], and the receiver parameters used are based on the actual system used in the aviation industry [28].

Table 3. Simulation parameters

\begin{tabular}{|c|c|c|}
\hline No. & Parameter & Value \\
\hline 1 & Transmit power & $250 \mathrm{Watt}$ \\
\hline 2 & Carrier frequency & $1090 \mathrm{MHz}$ \\
\hline
\end{tabular}




\begin{tabular}{|c|c|c|}
\hline 3 & GRS receiver sensitivity & $-90 \mathrm{dBm}$ \\
\hline 4 & GRS antenna gain & $12 \mathrm{dBi}$ \\
\hline 5 & Transmitter antenna gain & $3 \mathrm{dBi}$ \\
\hline
\end{tabular}

\section{B. Simulation Results and Discussion}

This section validates the performance model with Monte Carlo simulation for the PE error. First, the effect of receiver separation on the PE error is estimated, followed by the relationship between the PDE error and the effective SNR for different sampling rates. Using the PDE error, the position error is derived for each position of emitter. Finally, Monte Carlo simulation verifies the position error obtained by the performance model.

\section{B.1. PE Error versus Receiver Separation}

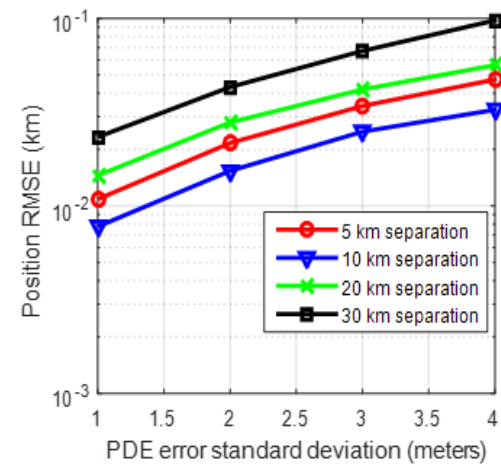

(a) Emitter range $5 \mathrm{~km}$

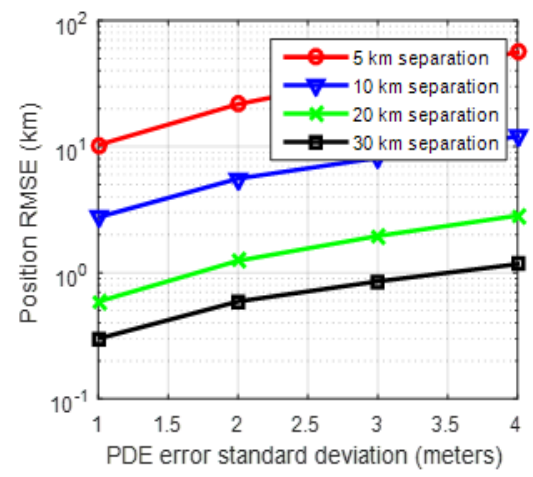

(c) Emitter range $100 \mathrm{~km}$

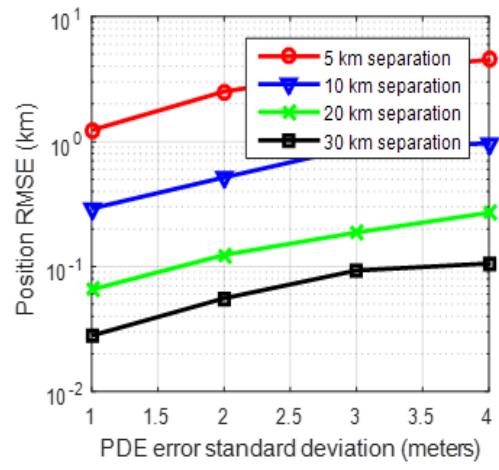

(b) Emitter range of $50 \mathrm{~km}$

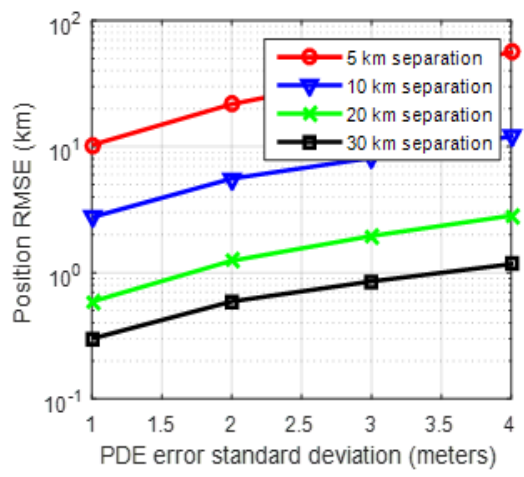

(d)Emitter range of $150 \mathrm{~km}$

Figure 2. PE error versus PDE error standard deviation for various GRS separation. [Emitter is at bearing of $60^{\circ}$ and altitude of $\left.1 \mathrm{~km}\right]$.

The PDE error modelled as a zero-mean Gaussian random variable is introduced into the PD in Eq. (4), and the PE is then estimated using Eq. (10) and Eq. (12). Signal attenuation due to path loss determines the received SNR. Monte Carlo simulations conducted based on 100 realizations for PDE error have a standard deviation range of 1 to 4 meters at selected positions of emitters with the range of $5 \mathrm{~km}, 50 \mathrm{~km}, 100 \mathrm{~km}$ and $150 \mathrm{~km}$ to determine the effect of GRS separation on the PE error. Figure 2 shows the PE error comparisons for different GRS separations at different positions of emitters. The PDE error standard deviation generally increases with the PE error. At an emitter range of $5 \mathrm{~km}$ and a PDE error of 2 meters, the PE error for GRS separations of $5 \mathrm{~km}, 10 \mathrm{~km}, 20 \mathrm{~km}$ and $30 \mathrm{~km}$ are $0.02 \mathrm{~km}, 0.015 \mathrm{~km}, 0.03 \mathrm{~km}$, and 
$0.04 \mathrm{~km}$, respectively. At an emitter range of $50 \mathrm{~km}$ and a PDE error of 2 meters, the PE error for the GRS separations are $2.51 \mathrm{~km}, 0.52 \mathrm{~km}, 0.12 \mathrm{~km}$, and $0.06 \mathrm{~km}$, respectively. For a shorter emitter range at less than $5 \mathrm{~km}$ and a large GRS separation of $30 \mathrm{~km}$, a high PE error of $0.04 \mathrm{~km}$ is obtained while a larger emitter range of $150 \mathrm{~km}$ produce a small PE error of $0.04 \mathrm{~km}$. On the average, the $10 \mathrm{~km}$ GRS separation always gives the best performance in term of PE error versus PDE error.

\section{B.2. PDE Error versus Receiver Sampling Frequency}

The TDOA measured between two signals is obtained from the peak of cross-correlation. Multiplying the measured TDOA with the speed of light in (2) estimates the PD. Factors that contribute to TDOA measurement errors are noise and sampling frequency. Based on 100realization Monte Carlo simulation, PDE error versus effective SNR comparison was made at the receiver sampling frequencies of $40 \mathrm{MHz}$ and $160 \mathrm{MHz}$. The existing software-defined radio (SDR) technologies available to be implemented forms the basis for selecting the sampling frequencies [27]. Figure 3 shows the PDE error versus effective SNR comparison for the two sampling frequencies. In general, the PDE error decreases exponentially with the effective SNR from $0 \mathrm{~dB}$ to $25 \mathrm{~dB}$. However, the PDE error is lower for the sampling frequency of $160 \mathrm{MHz}$ compared to $40 \mathrm{MHz}$. At an effective SNR of $4 \mathrm{~dB}$, the resulting PDE errors for receiver sampling frequencies of $40 \mathrm{MHz}$ and $160 \mathrm{MHz}$, are 30.13 meters and 14.56 meters respectively while at an effective SNR of $18 \mathrm{~dB}$ the PDE error values are 1.7 meters and 0.88 meters. The actual relationship between the SNR and PDE errors very much depends on the algorithms to estimate the TDOA. Various methods for TDOA estimation are described in $[2,9,11]$.

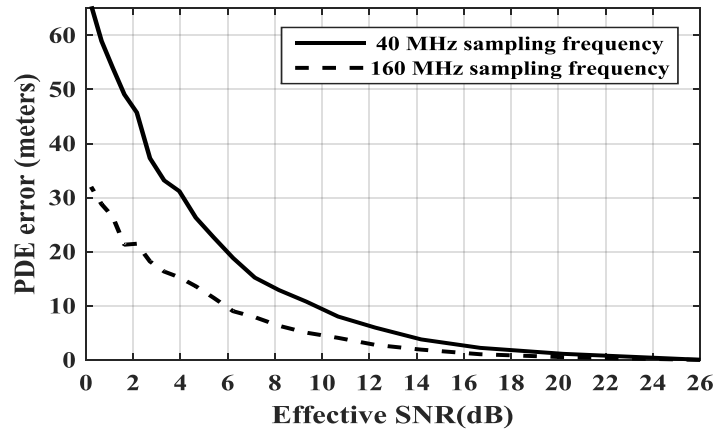

Figure 3. Cross correlation based PDE error versus effective SNR

Using curve fitting techniques, a mathematical equation for the relationship between the PDE error and the effective SNR is derived. The following equation describes the relationship between the PDE error $(\Delta d)$ and the effective SNR $\left(S N R_{e f f}\right)$ for the sampling frequency of $160 \mathrm{MHz}$

$$
\Delta d_{\text {eff }}= \begin{cases}32.6 \times \exp \left(-0.1984 \times S N R_{\text {eff }}\right) & 0 \leq S N R_{\text {eff }} \leq 25 \\ 0 & S N R_{\text {eff }}>25\end{cases}
$$

Thus, the PDE error can be estimated for a range of effective SNR.

\section{B.3. PE Error Comparison}

Using the PDE error obtained from the previous section for different positions of emitter based on the GRS separation of $10 \mathrm{~km}$ and the sampling frequency of $160 \mathrm{MHz}$, Monte Carlo simulation compares the performance model in terms of horizontal PE error and altitude PE error at 500 realizations for emitter range and bearing at intervals of $5 \mathrm{~km}$ and $7.5^{0}$ respectively. The selected intervals are to reduce the computational time of Monte Carlo simulation and to ensure sufficient results for comparison. The GRS 1 and GRS 2 when used as the GRS reference pair 
results in blind spots along bearings of emitter at $0^{\circ}, 90^{\circ}, 135^{\circ}, 180^{\circ}, 270^{\circ}$ and $315^{\circ}$, which are indicated by the black dash line in the plots.

\section{a. Horizontal PE error}

Figure 4 compares the horizontal PE errors obtained using the performance model defined by Eq. (27) with that of Monte Carlo simulation results. The comparison between Monte Carlo and the performance model shows that the horizontal PE accuracy of emitter depends on the position of emitter. The horizontal PE error increases exponentially with an increase in the emitter range from $1 \mathrm{~km}$ to $200 \mathrm{~km}$. The absolute error of the performance model is compared with the Monte Carlo simulation results. The performance model for horizontal PE error defined by Eq. (27) predicts the error in the horizontal position of emitter is compared to the Monte Carlo simulation with an absolute error difference of $0.1 \mathrm{~km}$ for an emitter range of $1 \mathrm{~km}$ to $200 \mathrm{~km}$ based on the GRS separation of $10 \mathrm{~km}$. Thus, the proposed horizontal PE performance model predicts the error in the horizontal position for a given PDE error with a prediction error of 0.1 km compared to the Monte Carlo simulation results.

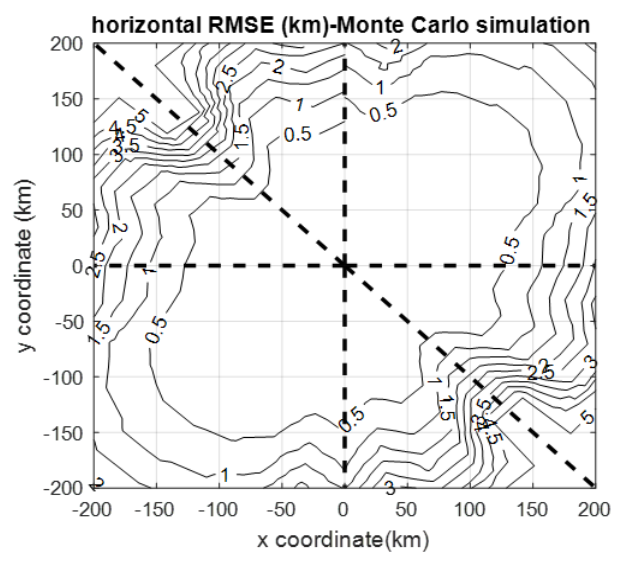

(a)

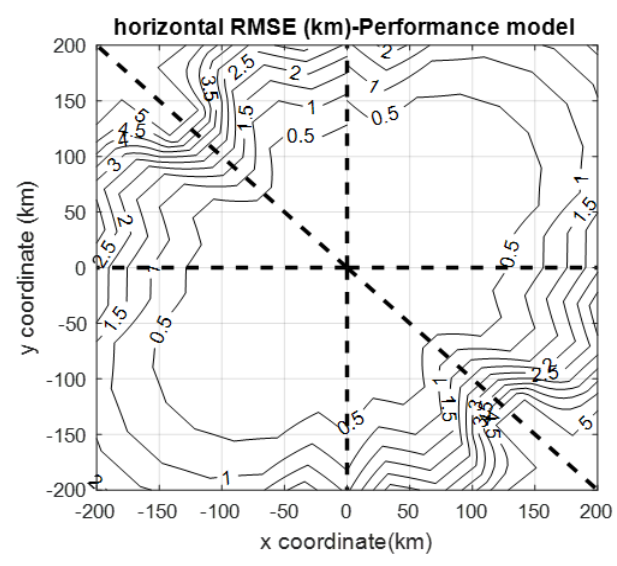

(b)

Figure 4. Horizontal PE error comparison between the performance model and the Monte Carlo simulation for emitter range up to $200 \mathrm{~km}$ [10 km GRS separation]: (a) Monte Carlo simulation, (b) Performance model.

\section{b. Altitude Estimation Error}

This section compares the altitude estimation error of the performance model defined by Eq. (31) with the Monte Carlo simulation results. Figure 5 shows the altitude estimation error based on the position of emitter shown in Table 1 using a GRS separation of $10 \mathrm{~km}$. The error in the altitude estimation depends on the altitude of emitter and the range of emitter. The altitude estimation error decreases with the increase in emitter range from $1 \mathrm{~km}$ to $200 \mathrm{~km}$ and the increase in altitude of emitter from $1 \mathrm{~km}$ to $15 \mathrm{~km}$. Table 4 summarizes the performance model for altitude estimation error at various altitudes. The performance model predicts the altitude error with a minimum absolute altitude error of $0.2 \mathrm{~km}$ at an altitude of emitter of $15 \mathrm{~km}$ up to a range of $200 \mathrm{~km}$ and a maximum absolute altitude error of $0.8 \mathrm{~km}$ at an altitude of $1 \mathrm{~km}$ up to a range of $100 \mathrm{~km}$.

Thus, the proposed performance model does not accurately predict the altitude error. This is due to the quadratic relation between the input and the output as seen in Eq. (31) which results in magnitude-squared error that decreases with the increase in altitude level.

Table 4. Altitude estimation error based on the performance model for various ranges and altitudes.

\begin{tabular}{|l|l|l|l|l|}
\hline No. & GRS & Altitude & Emitter & Absolute Altitude \\
\hline
\end{tabular}




\begin{tabular}{|c|c|c|c|c|}
\hline & $\begin{array}{c}\text { separation } \\
(\mathrm{km})\end{array}$ & $\begin{array}{c}\text { levels } \\
\text { (km) }\end{array}$ & $\begin{array}{c}\text { Range } \\
\text { (km) }\end{array}$ & error (km) \\
\hline \multirow{2}{*}{1} & \multirow{3}{*}{10} & 1 & 0 to 100 & 0.8 \\
\cline { 1 - 3 } & & 7 & 0 to 200 & 0.5 \\
\cline { 3 - 5 } 3 & & 15 & 0 to 200 & 0.2 \\
\hline
\end{tabular}
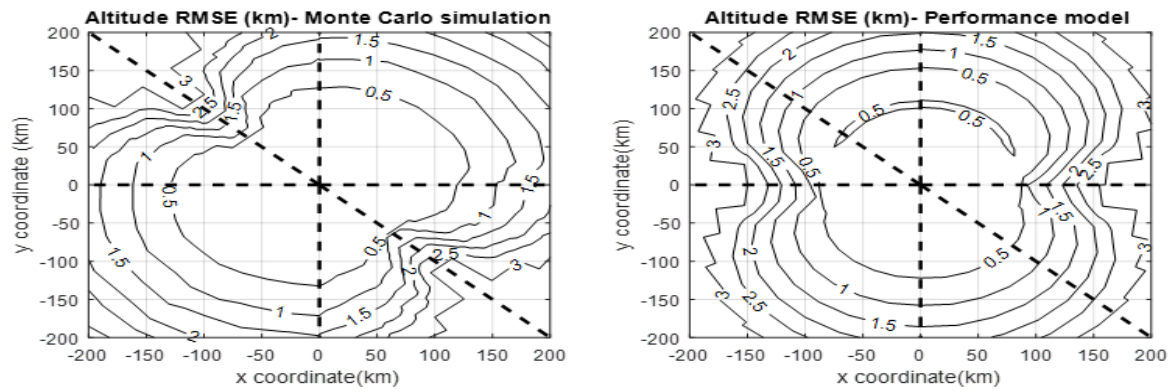

(a) $1 \mathrm{~km}$ emitter altitude
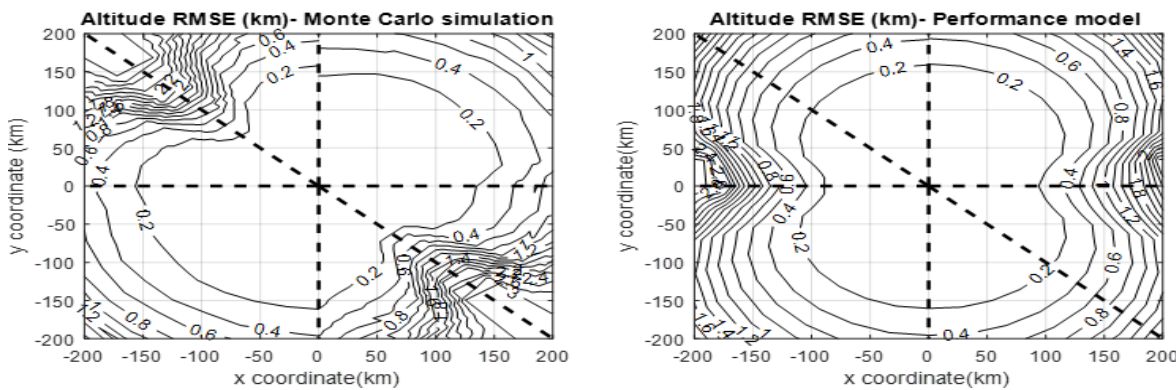

(b) $7 \mathrm{~km}$ emitter altitude
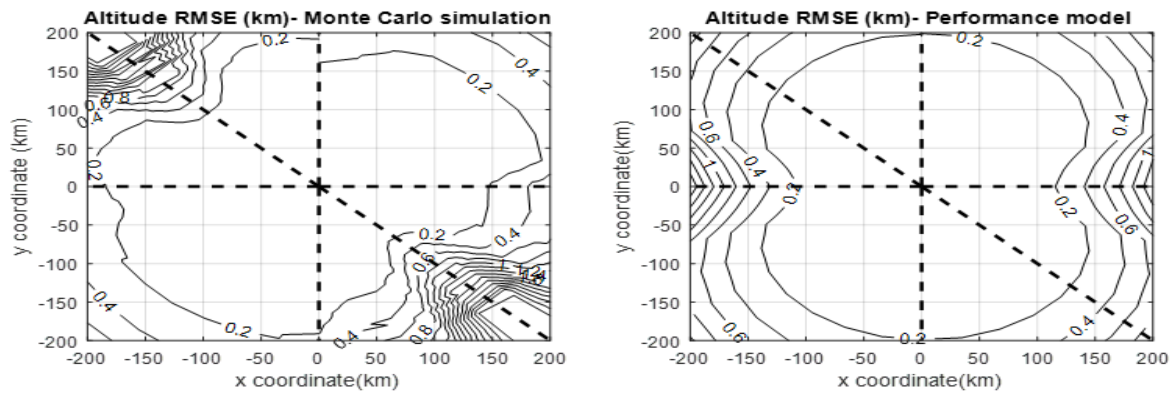

(c) $15 \mathrm{~km}$ emitter altitude

Figure 5. Comparison between performance model with Monte Carlo simulation for altitude estimation error at $10 \mathrm{~km}$ GRS separation.

\section{Conclusion}

A 3-D multiple-reference multilateration algorithm using minimum GRS is proposed and the performance model for PE error is developed. A square GRS configuration is assumed for the performance model for emitters locating within a bearing of $360^{\circ}$, with a range of up to $200 \mathrm{~km}$ and a maximum altitude of $15 \mathrm{~km}$. The characteristics of emitters are based on the ADS-B transponder used in civil aviation. Verification of the model with Monte Carlo simulation shows that the performance model estimates the maximum horizontal PE error of $0.1 \mathrm{~km}$ for a range of $200 \mathrm{~km}$ and an altitude error of $0.2 \mathrm{~km}$ at an altitude of $15 \mathrm{~km}$. The performance model also predicts the blind spots in the emitter locating coverage. Thus, the performance model predicts the horizontal PE error and the error at high altitudes for a minimum configuration 
multilateration system with a square GRS configuration. However, the performance model does not provide the optimal solution that minimizes the PE error.

\section{Acknowledgments}

The authors thank Universiti Teknologi Malaysia (UTM) for providing the resources and support for this research.

\section{References}

[1]. M. A. C. Duran et al., "Terrestrial Network-Based Positioning and Navigation," in Satellite and Terrestrial Radio Positioning Techniques, 2012, pp. 75-153.

[2]. W. H. L. Neven, T. J. Quilter, R. Weedo, and R. A. Hogendoorn, "Wide area multilateration (WAM)," Report on EATMP TRS 131/04 Version 1.1, 2005.

[3]. R. Zekavat and R. M. Buehrer, Handbook of position location: Theory, practice and advances. John Wiley \& Sons, Inc., 2012.

[4]. ICAO, "Guidance Material on Comparison of Surveillance Technologies (GMST)," Montreal, Quebec, 2001.

[5]. Y. Wang, G. Leus, and H. Deliç, "Time-of-arrival estimation by UWB radios with low sampling rate and clock drift calibration," Signal Processing, vol. 94, pp. 465-475, Jan. 2014.

[6]. L. Blanco, J. Serra, and M. Nájar, "Minimum variance time of arrival estimation for positioning," Signal Processing, vol. 90, no. 8, pp. 2611-2620, Aug. 2010.

[7]. G. Galati, M. Leonardi, P. Magaro, and V. Paciucci, "Wide Area Surveillance Using SSR Mode S Multilateration: Advantages and Limitations," in European Radar Conference, 2005. EURAD 2005., 2005, pp. 245-250.

[8]. K. Haneda, K. Takizawa, J. Takada, M. Dashti, and P. Vainikainen, "Performance evaluation of threshold-based UWB ranging methods: Leading edge vs. search back," in Proc. of EuCAP'09 - 3rd IEEE European Conference on Antennas and Propagation, 2009, pp. 3673-3677.

[9]. J. Falk, "An electronic warfare perspective on time difference of arrival estimation subject to radio receiver imperfections," 2004.

[10]. H. Marmaroli, Patrick; Falourd, Xavier; Lissek, "A Comparative Study of Time Delay Estimation Techniques for Road Vehicle Tracking," in Proceedings of the 11th French Congress of Acoustics and 2012 Annual IOA Meeting, 2012.

[11]. Knapp and G. Carter, "The generalized correlation method for estimation of time delay," IEEE Transactions on Acoustics, Speech, and Signal Processing, vol. 24, no. 4, pp. 320 327, Aug. 1976.

[12]. I. A. Mantilla-Gaviria, M. Leonardi, G. Galati, and J. V. Balbastre-Tejedor, "Localization algorithms for multilateration (MLAT) systems in airport surface surveillance," Signal, Image and Video Processing, vol. 9, no. 7, pp. 1549-1558, Oct. 2015.

[13]. G. Galati, M. Leonardi, J. V. Balbastre-Tejedor, and I. A. Mantilla-Gaviria, "Timedifference-of-arrival regularised location estimator for multilateration systems," IET Radar, Sonar \& Navigation, vol. 8, no. 5, pp. 479-489, Jun. 2014.

[14]. W. Lei and B. Chen, "High-precision hyperboloid location method using passive timedifference-of-arrival measurements," IET Radar, Sonar \& Navigation, vol. 7, no. 6, pp. 710-717, 2013.

[15]. D. E. Chaitanya, M. N. V. S. S. Kumar, G. S. Rao, and R. Goswami, "Convergence issues of taylor series method in determining unknown target location using hyperbolic multilateration," 2014 International Conference on Science Engineering and Management Research, ICSEMR 2014, no. 1, pp. 1-4, 2015.

[16]. D.-G. Kim, Y.-H. Kim, and H.-N. Kim, "Two-step estimator for moving-emitter geolocation using time difference of arrival/frequency-difference of arrival measurements," IET Radar, Sonar \& Navigation, vol. 9, no. 7, pp. 881-887, 2015. 
[17]. H. Torbati Fard, M. Atashbar, Y. Norouzi, and F. Hojjat Kaskani, "Multireference TDOAbased source localization," Turkish Journal of Electrical Engineering \& Computer Sciences, vol. 21, pp. 1920-1929, 2013.

[18]. A. Z. Sha'ameri, Y. A. Shehu, and W. Asuti, "Performance Analysis of a Minimum Configuration Multilateration System for Airborne Emitter Position Estimation," Defence S and T Technical Bulletin, vol. 8, no. 1, pp. 27-41, 2015.

[19]. M. Stojilovi, B. Menssen, I. Flintoft, H. Garbe, J. Dawson, and M. Rubinstein, "TDoAbased localisation of radiated IEMI sources," IEEE International Symposium on Electromagnetic Compatibility, pp. 1263-1268, 2014.

[20]. K. C. Ho, "Bias reduction for an explicit solution of source localization using TDOA," IEEE Transactions on Signal Processing, vol. 60, no. 5, pp. 2101-2114, 2012.

[21]. H. W. L. Naus, "Analytical geolocation and three-dimensional localisation by time difference of arrival," IET Radar, Sonar \& Navigation, vol. 10, no. 9, pp. 1643-1649, Dec. 2016.

[22]. R. Bucher and D. Misra, "A Synthesizable VHDL Model of the Exact Solution for Threedimensional Hyperbolic Positioning System," VLSI Design, vol. 15, no. 2, pp. 507-520, 2002.

[23]. M. D. Gillette and H. F. Silverman, "A linear closed-form algorithm for source localization from time-differences of arrival," IEEE Signal Processing Letters, vol. 15, no. 1, pp. 1-4, 2008.

[24]. J. Y. Huang, Q. Wan, and P. Wang, "Minimum mean square error estimator for mobile location using time-difference-of-arrival measurements," IET Radar, Sonar \& Navigation, vol. 5, no. 2, p. 137, 2011.

[25]. M. A. Sebt and A. Noroozi, "Weighted least squares target location estimation in multitransmitter multi-receiver passive radar using bistatic range measurements," IET Radar, Sonar \& Navigation, vol. 10, no. 6, pp. 1088-1097, 2016.

[26]. ICAO, "Multilateration (MLAT) concept of use: Edition 1.0 September 2007," 2007.

[27]. SELEX Sistemi Integrati, “ADS-B Subsystem: Standard E5010015201SDD,” 2014.

[28]. ERA, "Vera-Ng Sees Without Being Seen." . [Online]. Available: http://www.era.cz/military-security/vera-ng. Accessed: Nov. 20, 2016.

[29]. SAAB, "Multilateration System High Performance Positioning on the Ground and in the Air.".

[30]. J. Zhou, J. Shi, and X. Qu, "Landmark placement for wireless localization in rectangularshaped industrial facilities," IEEE Transactions on Vehicular Technology, vol. 59, no. 6, pp. 3081-3090, 2010.

[31]. Y. Chen, J. Francisco, W. Trappe, and R. Martin, "A Practical Approach to Landmark Deployment for Indoor Localization," in 2006 3rd Annual IEEE Communications Society on Sensor and Ad Hoc Communications and Networks, 2006, pp. 365-373.

[32]. A. Bais, H. Kiwan, and Y. Morgan, "On Optimal Placement of Short Range Base Stations for Indoor Position Estimation," Journal of Applied Research and Technology, vol. 12, no. October, pp. 886-897, 2014.

[33]. Y. Trofimova, "Multilateration Error Investigation and Classification. Error Estimation," Transport and Telecommunication, vol. 8, no. 2, pp. 28-37, 2007.

[34]. I. A. Mantilla-Gaviria, M. Leonardi, G. Galati, J. V. Balbastre-Tejedor, and E. de los Reyes Davó, "An effective procedure to design the layout of standard and enhanced mode-S multilateration systems for airport surveillance," International Journal of Microwave and Wireless Technologies, vol. 4, no. 2, pp. 199-207, Apr. 2012.

[35]. G. H. Golub and C. F. Van Loan, Matrix Computations. 2013.

[36]. M. Geyer, "Aircraft Navigation and Surveillance Analysis for a Spherical Earth," Project Memorandum, DOT-VNTSC-FAA-15-01, Prepared for FAA-WTRO, no. October 2014, 2014.

[37]. R. Francis et al., "The Flying Laboratory for the Observation of ADS-B Signals," International Journal of Navigation and Observation, vol. 2011, no. 2011, pp. 1-5, 2011. 
Appendix A: Derivation of relative GRS geometry and PD matrix error as a function of PDE error and $P D$ measurement matrix

This appendix discusses on how the relative GRS geometry and PD matrix error $\left(\frac{\|\Delta \mathbf{A}\|_{2}}{\|\mathbf{A}\|_{2}}+\frac{\|\Delta \mathbf{b}\|_{2}}{\|\mathbf{b}\|_{2}}\right)$ is expressed as a function of PDE error $(\Delta \boldsymbol{d})$ and PD measurement matrix. Let the coordinates of GRS 1, GRS 2, GRS 3 and GRS 4 in rectangular coordinate system be $\left(\mathbf{x}_{1}, \mathbf{y}_{1}, \mathbf{z}_{1},\right),\left(\mathbf{x}_{2}, \mathbf{y}_{2}, \mathbf{z}_{2},\right),\left(\mathbf{x}_{3}, \mathbf{y}_{3}, \mathbf{z}_{3},\right)$ and $\left(\mathbf{x}_{4}, \mathbf{y}_{4}, \mathbf{z}_{4},\right)$ respectively. From Table 2 in Section 5.1 , the following equations can be derived for the square ground receiving station (GRS) configuration:

$$
\begin{aligned}
& x_{1}=-0.5 \times d_{r e c}, x_{2}=0.5 \times d_{r e c} x_{3}=0.5 \times d_{r e c}, x_{4}=-0.5 \times d_{r e c} \\
& y_{1}=0.5 \times d_{r e c}, y_{2}=-0.5 \times d_{r e c}, \quad y_{3}=0.5 \times d_{r e c}, y_{4}=-0.5 \times d_{r e c}
\end{aligned}
$$

where $d_{\text {rec }}$ is the GRS pair separation.

Substituting (7) into matrix (10a), using GRS 1 and GRS 2 as reference pair, the entries of matrix Q are

$$
\mathbf{Q}=\left[\begin{array}{ll}
\left(\frac{x_{3}-x_{1}}{d_{13}}-\frac{x_{4}-x_{1}}{d_{14}}\right) & \left(\frac{y_{3}-y_{1}}{d_{13}}-\frac{y_{4}-y_{1}}{d_{14}}\right) \\
\left(\frac{x_{3}-x_{2}}{d_{23}}-\frac{x_{4}-x_{2}}{d_{24}}\right) & \left(\frac{y_{3}-y_{2}}{d_{23}}-\frac{y_{4}-y_{2}}{d_{24}}\right)
\end{array}\right]
$$

and matrix a

$$
\mathbf{a}=0.5 \times\left[\begin{array}{l}
\left(d_{14}-d_{13}+\frac{K_{1}-K_{4}}{d_{14}}-\frac{K_{1}-K_{3}}{d_{13}}\right) \\
\left(d_{24}-d_{23}+\frac{K_{2}-K_{4}}{d_{24}}-\frac{K_{2}-K_{3}}{d_{23}}\right)
\end{array}\right]
$$

where

$$
K_{i}=\left(x_{i}\right)^{2}+\left(y_{i}\right)^{2}+\left(z_{i}\right)^{2} \text { for } i \epsilon[1,2,3,4]
$$

Substituting Eq. (A.1) into Eq. (A.2), the matrix Q can be written as a function of $d_{r e c}$ and PD measurements. That is

$$
\begin{aligned}
\mathbf{Q} & =\left[\begin{array}{ll}
\left(\frac{0.5 d_{r e c}+0.5 d_{r e c}}{d_{13}}-\frac{-0.5 d_{r e c}+0.5 d_{r e c}}{d_{14}}\right) & \left(\frac{0.5 d_{r e c}-0.5 d_{r e c}}{d_{13}}-\frac{-0.5 d_{r e c}-0.5 d_{r e c}}{d_{14}}\right) \\
\left(\frac{0.5 d_{r e c}-0.5 d_{r e c}}{d_{23}}-\frac{-0.5 d_{r e c}-0.5 d_{r e c}}{d_{24}}\right) & \left(\frac{0.5 d_{r e c}+0.5 d_{r e c}}{d_{23}}-\frac{-0.5 d_{r e c}+0.5 d_{r e c}}{d_{24}}\right)
\end{array}\right] \\
& =\left[\begin{array}{ll}
\left(\frac{0.5 d_{r e c}+0.5 d_{r e c}}{d_{13}}\right) & -\left(\frac{-0.5 d_{r e c}-0.5 d_{r e c}}{d_{14}}\right) \\
-\left(\frac{-0.5 d_{r e c}-0.5 d_{r e c}}{d_{24}}\right) & \left(\frac{0.5 d_{r e c}+0.5 d_{r e c}}{d_{23}}\right)
\end{array}\right]
\end{aligned}
$$




$$
\begin{aligned}
& =\left[\begin{array}{ll}
\left(\frac{d_{r e c}}{d_{13}}\right) & \left(\frac{d_{r e c}}{d_{14}}\right) \\
\left(\frac{d_{r e c}}{d_{24}}\right) & \left(\frac{d_{r e c}}{d_{23}}\right)
\end{array}\right] \\
& =d_{r e c} \times\left[\begin{array}{ll}
\left(d_{13}\right)^{-1} & \left(d_{14}\right)^{-1} \\
\left(d_{24}\right)^{-1} & \left(d_{23}\right)^{-1}
\end{array}\right]
\end{aligned}
$$

Eq. (A.5d) is the representation of matrix Q as a function of the PD measurement and GRS pair separation. Substituting Eq. (A.1) into Eq. (A.3), the matrix a of Eq. (A.3) can be further simplified to:

$$
\mathbf{a}=0.5 \times\left[\begin{array}{l}
\left(d_{14}-d_{13}\right) \\
\left(d_{24}-d_{23}\right)
\end{array}\right]
$$

Since

$$
\begin{aligned}
& K_{1}-K_{4}=\left(x_{1}\right)^{2}-\left(x_{4}\right)^{2}+\left(y_{1}\right)^{2}-\left(y_{4}\right)^{2}+\left(z_{1}\right)^{2}-\left(z_{4}\right)^{2}=0 \\
& K_{1}-K_{3}=\left(x_{1}\right)^{2}-\left(x_{3}\right)^{2}+\left(y_{1}\right)^{2}-\left(y_{3}\right)^{2}+\left(z_{1}\right)^{2}-\left(z_{3}\right)^{2}=0 \\
& K_{2}-K_{4}=\left(x_{2}\right)^{2}-\left(x_{4}\right)^{2}+\left(y_{2}\right)^{2}-\left(y_{4}\right)^{2}+\left(z_{2}\right)^{2}-\left(z_{4}\right)^{2}=0 \\
& K_{2}-K_{3}=\left(x_{2}\right)^{2}-\left(x_{3}\right)^{2}+\left(y_{2}\right)^{2}-\left(y_{3}\right)^{2}+\left(z_{2}\right)^{2}-\left(z_{3}\right)^{2}=0
\end{aligned}
$$

Assuming there is an error in the PD measurement, the perturbed version of matrix Q is obtained as:

$$
\hat{\mathbf{Q}}=d_{r e c} \times\left[\begin{array}{ll}
\left(d_{13}+\Delta d_{\text {eff }}\right)^{-1} & \left(d_{14}+\Delta d_{\text {eff }}\right)^{-1} \\
\left(d_{24}+\Delta d_{e f f}\right)^{-1} & \left(d_{13}+\Delta d_{e f f}\right)^{-1}
\end{array}\right]
$$

where $\Delta d_{\text {eff }}$ is the PDE error that corresponds to the effective SNR which is obtained using (33) and that of matrix a is:

$$
\hat{\mathbf{a}}=0.5 \times\left[\begin{array}{l}
\left(d_{14}+\Delta d\right)-\left(d_{13}+\Delta d\right) \\
\left(d_{24}+\Delta d\right)-\left(d_{23}+\Delta d\right)
\end{array}\right] \approx 0.5 \times\left[\begin{array}{l}
d_{14}-d_{13} \\
d_{24}-d_{23}
\end{array}\right]
$$

The error in matrix $\mathrm{Q}$ due to $\Delta d$ is calculated as:

$$
\begin{aligned}
\Delta \mathbf{Q}=\mathbf{Q}-\hat{\mathbf{Q}} & =d_{r e c} \times\left(\left[\begin{array}{ll}
d_{13}^{-1} & d_{14}{ }^{-1} \\
d_{24}^{-1} & d_{23}{ }^{-1}
\end{array}\right]-\left[\begin{array}{ll}
\left(d_{13}+\Delta d\right)^{-1} & \left(d_{14}+\Delta d\right)^{-1} \\
\left(d_{24}+\Delta d\right)^{-1} & \left(d_{23}+\Delta d\right)^{-1}
\end{array}\right]\right) \\
& =d_{r e c} \times \Delta d \times\left[\begin{array}{ll}
d_{13}^{-2} & d_{14}^{-2} \\
d_{24}^{-2} & d_{23}^{-2}
\end{array}\right]
\end{aligned}
$$

The error in matrix a due to $\Delta d$ is calculated as:

$$
\Delta \mathbf{a}=\mathbf{a}-\hat{\mathbf{a}}=\left[\begin{array}{l}
d_{14}-d_{13} \\
d_{24}-d_{23}
\end{array}\right]-\left[\begin{array}{l}
d_{14}-d_{13} \\
d_{24}-d_{23}
\end{array}\right]=0
$$

The relative error in matrix $\mathrm{Q},\left(\frac{\|\Delta \mathbf{Q}\|_{2}}{\|\mathbf{Q}\|_{2}}\right)$ is obtained as: 


$$
\left(\frac{\|\Delta \mathbf{Q}\|_{2}}{\|\mathbf{Q}\|_{2}}+\frac{\|\Delta \mathbf{a}\|_{2}}{\|\mathbf{a}\|_{2}}\right)=\Delta d \times\left\|\left[\begin{array}{ll}
d_{13}^{-2} & d_{14}^{-2} \\
d_{24}^{-2} & d_{23}^{-2}
\end{array}\right]\right\|_{2} \times\left\|\left[\begin{array}{ll}
d_{13}^{-1} & d_{14}{ }^{-1} \\
d_{24}^{-1} & d_{23}{ }^{-1}
\end{array}\right]^{-1}\right\|_{2}
$$

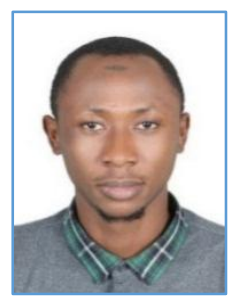

Abdulmalik S. YARO was born in Kaduna, Nigeria. He received his B.Eng. degree in Electrical Engineering from Ahmadu Bello University, Zaria in 2012 and his M. Eng. degree in Electrical-Electronics and Telecommunications from University Teknologi Malaysia in 2014. He is currently pursuing his Ph.D. degree on Surveillance system at the Department of Electrical and Computer Engineering at Universiti Teknologi Malaysia.

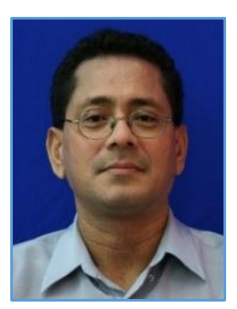

Ahmad Z. SHA'AMERI obtained his B. Sc. in Electrical Engineering from the University of Missouri-Columbia, USA in 1984, and M. Eng. Electrical Engineering and $\mathrm{PhD}$ both from UTM in 1991 and 2000 respectively. At present, he is an associate professor, Coordinator for the Digital Signal and Image Processing (DSIP) Research Group and Academic Coordinator for the DSP Lab, Electronic and Computer Engineering Department, Faculty of Electrical Engineering, UTM. His research interest includes signal theory, signal processing for radar and communication, signal analysis and classification, and information security. The subjects taught at both undergraduate and postgraduate levels include digital signal processing, advance digital signal processing, advance digital communications and information security. He has also conducted short courses for both government and private sectors. At present, he has published 160 papers in his areas of interest at both national and international levels in conferences and journals.

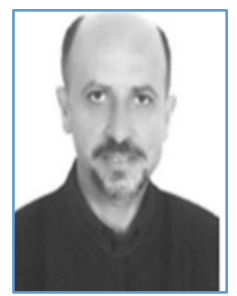

Nidal KAMIL obtained the PhD degree (Hons) from the Technical University of Gdansk, Poland, in 1993. Since 1993 he has been involved in research projects related to estimation theory, noise reduction, optimal filtering, and pattern recognition. $\mathrm{He}$ developed SNR estimation for antenna diversity combining, single-trial subspacebased technique for ERP extraction from brain background noise, time-constraints optimization technique for speckle noise reduction in SAR images, and introduced data glove for online signature verification. His present research interest is mainly in EEG signal processing for localization of brain sources, assessment of cognitive and visual distraction, Neuro feedback, learning and memory recall, in addition to fMRI- EEG data fusion. He is the editor of EEG/ERP Analysis: Methods and Applications, CRC Press, NY, 2015. Currently, he is Associate Professor at the PETRONAS University of Technology, Malaysia. 